\title{
Characterizing the Stages of Complex Tasks
}

\author{
Jiqun Liu \\ School of Communication and Information, Rutgers University \\ New Brunswick, New Jersey \\ j12033@scarletmail.rutgers.edu
}

\begin{abstract}
Stage is an essential facet of task. At different stages of search, users' search strategies are often influenced by different search intentions, encountered problems, as well as knowledge states. In previous studies, information seeking and interactive IR researchers have developed and validated some frameworks for describing various task facets and features. However, few studies have explored how to depict and differentiate different stages or states of complex search tasks in a comprehensive, multidimensional manner. The existing theoretical models of search process offer limited contributions to search path evaluation and the design of system recommendations for users at different states. To address this issue at both theoretical and empirical levels, my dissertation aims to construct an explainable framework that can characterize the stages or states of complex search tasks over multiple dimensions and to apply the framework in proactive search path evaluation and recommendation.
\end{abstract}

\section{CCS CONCEPTS}

\section{- Information systems $\rightarrow$ Users and interactive retrieval.}

\section{KEYWORDS}

Complex task; Multidimensional task stage; Information searching

\section{MOTIVATION}

Tasks of different types often emerge from evolving, continuous problematic situations when people interact with various elements embedded in their life-worlds. Search systems and technologies have experienced phenomenal success in recent years, especially in addressing fact-finding and navigational search tasks [6]. However, current search systems, interaction models, and the underlying algorithms still face plenty of challenges when applied in supporting complex tasks which involve multi-round, multidimensional search interactions and iterations (e.g., planning a research project, evaluating retirement packages) [1]. One of the major challenges in facilitating complex task is understanding and supporting the implicit task stages or states in search interactions.

\section{RESEARCH QUESTIONS AND METHODS}

The ultimate goal of this dissertation is to establish a multidimensional framework that can (1) characterize the stages or states of complex search tasks and (2) facilitate the evaluation of search

Permission to make digital or hard copies of part or all of this work for personal or classroom use is granted without fee provided that copies are not made or distributed for profit or commercial advantage and that copies bear this notice and the full citation on the first page. Copyrights for third-party components of this work must be honored

For all other uses, contact the owner/author(s).

SIGIR '19, July 21-25, 2019, Paris, France

(C) 2019 Copyright held by the owner/author(s)

ACM ISBN 978-1-4503-6172-9/19/07 ..\$15.00

https://doi.org/10.1145/3331184.3331413 actions and strategies as well as proactive search paths recommendations. Specifically, based on our recent research progresses on users' intentions, problems, and task-based search interactions (e.g., [2-4]), this work seeks to answer the following questions:

- RQ1 What are the stages of complex search tasks in terms of query-level search intentions, encountered problems, and search tactics?

- RQ2 What are the relations between different task stages?

- RQ3 How can we evaluate a search path using the multidimensional task stage framework?

- RQ4 How can we proactively offer useful system supports before actual search problems and difficulties happen?

Given the nature of the above research questions, this dissertation work consists of two parts. The first part includes two controlled lab studies where I controlled task types and collected data on multiple dimensions of search from users (e.g., search intentions, encountered problems and preferred helps, search actions), aiming to characterize task stages from different perspectives and also to understand the underlying connections among various states (RQ1 \& RQ2). A multidimensional stage framework of complex task will be constructed based on the findings from user studies as well as the relevant constructs from existing literature (e.g., Human problem solving [5]). The second part is a large scale experiment where I will run simulations based on the data collected from existing user studies and large-scale search logs and iteratively evaluate search paths as "users' policies in uncertain environments" using Reinforcement Learning approach. The proposed task stage framework will be employed to characterize the states, actions, and rewards in search paths. Finally, I will approach RQ4 as a policy optimization problem for unknown Markov Decision Process (MDP) and evaluate the quality of improved policies as (simulated) recommendations.

\section{ACKNOWLEDGEMENT}

I would like to thank my advisor, Dr. Chirag Shah. This work is supported by National Science Foundation grant IIS-1717488.

\section{REFERENCES}

[1] J Shane Culpepper, Fernando Diaz, and Mark D Smucker. 2018. Research Frontiers in Information Retrieval: Report from the Third Strategic Workshop on Information Retrieval in Lorne (SWIRL 2018). In ACM SIGIR Forum, Vol. 52. ACM, 34-90.

[2] Jiqun Liu. 2017. Toward a unified model of human information behavior: An equilibrium perspective. Fournal of Documentation 73, 4 (2017), 666-688.

[3] Jiqun Liu, Matthew Mitsui, Nicholas J. Belkin, and Chirag Shah. 2019. Task,information seeking intentions, and user behavior: Toward a multi-level understanding of Web search. In Proceedings of CHIIR 2019. ACM.

[4] Jiqun Liu, Yiwei Wang, Soumik Mandal, and Chirag Shah. 2019. Exploring the immediate and short-term effects of peer advice and cognitive authority on Web search behavior. Information Processing and Management 56, 3 (2019), 1010-1025.

[5] Allen Newell, Herbert Alexander Simon, et al. 1972. Human problem solving. Vol. 104. Prentice-Hall Englewood Cliffs, NJ.

[6] Ryen White. 2016. Interactions with search systems. Cambridge University Press. 